\title{
JARG blog
}

\section{Has PGD become too fashionable?}

Preimplantation genetic diagnosis (PGD) initially developed with the primary purpose of preventing genetic diseases in offspring. Few then probably anticipated that this highly complex technique 1 day would be used to improve IVF pregnancy rates through embryo selection (based on aneuploidy screening). European colleagues coined two separate acronyms for these "new" indications of PGD: PGD-AS, ${ }^{1}$ for preimplantation genetic diagnosis for aneuploidy screening and $P G S{ }^{2}$ for preimplantation genetic screening.

Aneuploidy screening has increasingly been offered under the quite logical assumption that the selection of euploid embryos, before embryo transfer, would lead to better pregnancy rates. Primary indications for PGD-AS/ PGS are advanced female age, repeated pregnancy loss and repeated IVF failure. Prominent investigators in the PGD arena, like Yuri Verlinsky, Ph.D and co-workers, from the Reproductive Genetics Institute in Chicago, IL, one of the world's leading PGD centers, have even argued for universal aneuploidy testing in all IVF cycles.

Follow up studies (though small in number) have, however, often failed to prove the concept and, starting at the Annual ASRM Meeting in New Orleans in November of 2006, the buzz in the hallways has been that these new indications for PGD may have to be seriously reevaluated. And the Europeans, once again, seem to have beaten us to the point. A rumor, circulating at the March 2007 World Congress on Controversies in Obstetrics and Gynecology in Barcelona, Spain, suggests that ESHRE was on the verge of publishing a position paper that calls into question the excessive utilization of PGD for any of these "newer" indications. Allegedly, it will go beyond the luke-warm statement of ESHRE Taskforce 5 on preimplantation genetic diagnosis. ${ }^{1}$

\footnotetext{
${ }^{1}$ This terminology was first utilized by ESHRE in the recently published Taskforce 5: preimplantation genetic diagnosis. In ESHRE Task Force on Ethics and law, ESHRE Monographs, ed. Maas Jan Heineman, Oxford University Press, January 2007.

${ }^{2}$ This terminology has been widely used in the European literature in the last few years and was also utilized by ESHRE in their annual PGD outcome reports.
}

For those who, however, really want to understand what is going on with PGD in aneuploidy testing, we strongly recommend the recently published paper by the almost always brilliant Jacques Cohen, Ph.D. (et al.) in Fertility Sterility (Cohen J et al., Fertil Steril 2007;87:496-503). Cohen in this paper, probably for the first time, presents a coherent concept about the utilization of PGD for aneuploidy testing, by acknowledging (contrary to widely propagated - though never documented - opinions of the "PGD-club") that embryo biopsy always, to some degree, reduces the embryo's implantation (and pregnancy) chances.

The fact that embryo biopsy inherently reduces the pregnancy chance from IVF, of course, not only represents a crucial acknowledgement, but a paradigm shift in PGD. As Cohen notes in his paper, it means that, if embryo selection by PGD is to lead to an improvement in IVF pregnancy rates through the selection of euploid embryos, then any potential benefit of PGD-AS/PGS has to outweigh the decrease in potential pregnancy chance from the embryo biopsy.

What Cohen is saying in practical terms is that, when considering PGD-AS/PGS we now in each case have to consider risk (decrease in pregnancy chance) versus potential benefit (improvement in overall pregnancy chance). In reviewing published data on embryo mass loss from cryopreservation, he suggests that removal of a single blastomere may reduce the embryo's pregnancy chance by approximately $12.5 \%$, while 2-cell removal would reduce the pregnancy chance by approximately $25 \%$. What Cohen's paper, therefore, really says is that the PGD-AS/PGSindications should be considered only very selectively after individualized risk/benefit considerations are properly evaluated.

Such considerations can be anticipated to lead to further paradigm shifts. For example, a 12.5 , or $25 \%$, decrease in pregnancy chance per embryo is more acceptable in cycles, where large embryo numbers are available, and less so with smaller numbers. In other words, a patient with only one or two embryos will be more affected than a woman with eight or nine embryos. Small embryo numbers are characteristically found in older women who, because of increasing aneuploidy rates, intuitively have been considered the best candidates for PGD-AS/PGS. Following Jacques Cohen's brilliant argument, they may turn out to be the worst. 


\section{Fashionably natural or fashionably mild}

Who hasn't noticed the recent infatuation with natural and/ or mild IVF cycles? Did you know that there now even exists a new International Society for Natural Cycle Assisted Reproduction, which recently held its 1st World (!) Congress in London, UK (First World Congress on natural/minimal stimulation IVF, London, Dec 15-16, 2006)? It is, to quote famous (baseball)"ART" expert, Yogi Bera, deja vu all over again for IVF old-timers. Haven't we been there already in the 1980s?

The question is whether there is real value in this new trend? This is, however, at least so-far, difficult to discern. Everybody, of course, would like to reduce medication usage, - if for no other reasons, than to reduce treatment costs for our patients [and, maybe, to get a little bit back at the not too popular pharmaceutical companies in our field (for some further detail, see below)]. Then there is also this widely held believe that high estrogen is detrimental to implantation [even though it seems probably equally likely that normalization of the estrogen/progesterone ratio in the luteal phase (through progesterone substitution) may avert this problem]. In short, it is "fashionable" to be "natural" and/or "mild." Al Gore, are you watching?

In reality, however, there is unfortunately only very little data in support of such approaches: First of all, the literature is confusing in even defining what should be considered a "natural" or "mildly stimulated" cycle and until we define what we really are talking about, it usually is better to say nothing.

This confusion seems to have carried over into reputable medical journals. For example, The Lancet recently published a paper from Prof. Fauser's well respected group at the Erasmus Medical Center in Utrecht, Netherlands (Heijnen et al., Lancet 2007;369:743-9), accompanied by an editorial by William L. Ledger, from the University of Sheffield, UK, (Ledger WL Lancet 2007;369:717-8), where a "mild treatment strategy" was defined $\mathrm{GnRH}$ antagonist co-treatment and single embryo transfer (s-ET), while patients were considered to receive "standard" treatment when a long agonist protocol was utilized with 2-embryo transfer (TET). Average gonadotropin (FSH) stimulation per day was 157.5 vs. 159.2 IU per day in "mild" and "standard" stimulations, respectively. Quite some mild stimulation! And since when does co-treatment by antagonist vs. agonist, or s-ET vs. TET, define "mild" stimulation?

The accompanying editorial by Ledger suggested that above noted Conference in London "rightly (and considering the topic of the Conference, not unsurprisingly) criticized the continued use of high-dose gonadotropins for IVF," and quite effusively applauded the Dutch study, which had demonstrated statistically comparable cumula- tive outcomes after their so-called "mild" and "standard" stimulations. Yet, neither authors, nor editorial commentator, ever make note of the fact that at study end, 12 months after randomization, only less than half of all women entering IVF treatment, had conceived a pregnancy that lead to live birth. While this may be considered "costeffective" (by authors and editorial commentator), without considering pregnancy outcomes, doing nothing could be seen as even more cost-effective!

Which brings us back to fashion: like any other field of human endeavor, medicine, is, of course, subject to fashion trends. The good news in all of this is, however, that every fashion is at some point destined to become passé.

\section{Fiction in medical journals?}

Something rather new happened in the March 29, 2007 issue of the New England Journal of Medicine, when Debora Spar, Ph.D, a Harvard Business School professor, opened the weekly issue of The Journal with a Perspective article, entitled: "The Egg Trade-Making Sense of the Market for Human Oocytes" (Spar D., N Engl J Med 2007;356:1289-1291).

She proceeded by introducing the readership to Anna Behrens, a 24 year old, tall and slim female, working towards a Ph.D. in art history (of course, at an Ivy League school), and heavily in debt, owing \$27,000. Over the ensuing paragraphs Anna eliminates her debt and acquires extra riches by becoming an egg donor (for which she is paid "\$20,000 plus medical expenses" in her 1st donation.). Shortly thereafter, now a "proven donor," she is paid $\$ 22,000$ for another donation, allowing her the luxury of a Caribbean vacation.

It takes five long paragraphs before the author reveals that "Anna Behrens is not a real person but a figment of her imagination." She then explains that she created this fictitious persona in an attempt to discuss some of the perceived irrationalities of what she considers a largely unregulated, human egg trade. The world's most famous medical journal has, thus, formally become publisher of (medical) fiction!

Dr. Spar's principal opinions about the commercialization of the "market for human oocytes" may, indeed, have value. The benefits of her opinion are, however, largely lost when her fiction turns into polemic: As a Harvard Business School professor and teacher, Dr. Spar, better than most, should understand that choice of words (and numbers) matters. When noting that oocyte donors "sell" their oocytes for an average of $\$ 5,000$ "per harvest," the connotation is obvious. She could, for example have described the process as oocyte donors receiving a $\$ 5,000$ average "reimbursement for their efforts" for each 
"retrieval." By raising egg donor reimbursement, in the fictional introduction of her assay, to an astronomical \$20,000-22,000 (plus medical expenses), she quite obviously takes considerable poetic liberties, since egg donors (except in out layer circumstances), of course, never receive such excessive payments.

And the purpose of all of this is? Who knows! It just seems surprising that the New England Journal of Medicine would lend its pages to such fictional polemics.

\section{No help from prayer}

Everybody, by now has probably accepted the sad fact that prayer, after all, does not improve IVF outcomes. For those few, who haven't heard this story yet,-yes, believe it, or not, in 2001 a medical journal (Journal of Reproductive Medicine, JRM) really published a "study" that claimed that prayers of people in the U.S., Canada and Australia doubled IVF pregnancy rates in Korea (-yes, it is again Korea, where, as we all recall, the first "successful" human cloning took place!)

Bruce Flamm, MD, a member of the Editorial Advisory Board of Ob.Gyn News, and professor at the University of California, Irvine, in the paper's March 15, 2007 issue offers a final summary of this, by now, legendary saga, involving Kwang Cha, M.D. (Korea), Roger Lobo, M.D. (Columbia University, New York) and Daniel Wirth (no degrees), the three authors of the original paper. The outtake of his article deserves exact quotation: "This may be the first time in history that all three authors of a randomized, controlled study have been found guilty of fraud, deception, and/or plagiarism."

Why the resurrection of this story, after such a long time? After all, already in May 2004 the $J R M$ had removed the study from its Internet site, after co-author Wirth had pleaded guilty to felony charges and acknowledged having "set up," and "managed" the various prayer groups. Coauthor Lobo, formally removed his name from the paper shortly after Wirth was in November 2005 sentenced to 5 years in federal prison. This left only the paper's 1st author, Cha, still formally linked to the paper. Nevertheless, $J R M$, quite surprisingly, returned the paper to its website.

The story now suddenly got legs again because Cha was just charged with plagiarism. According to a
February 18, 2007 article in the Los Angeles Times, and confirmed by Alan DeCherney, MD, Editor-in Chief of Fertility Sterility, an article by Cha et al. in Fertility and Sterility in 2005 "was a word-for-word, chart-for-chart, copy of a paper previously published by a different author in a Korean medical journal."

Understandably, Flamm wants the original paper finally formally removed from, what he calls, the "legitimate peerreviewed literature." Who can blame him? But, what an ending to an almost holy story!

\section{Short stories}

It may be, after all, worthwhile taking rumors, reported here in the pages of the JARG-Blog, seriously. Two such rumors can now be confirmed: When, Akzo Nobel late in 2006, and concomitantly with the sale of Serono to Merck KGaA (now combined Merck-Serono) announced the reorganization of Organon BioSciences into an independent subsidiary, we predicted that this was only a prelude to the sale of the company. On March 12, 2007 Akzo Nobel now, indeed, announced the sale of Organon to Schering-Plough for a whopping 11 billion in cash (multiply by 1.35 for dollar value). Considering these incredibly high valuations, how far can Ferring Pharmaceuticals be behind?

We also reported the rumor that our editorial board member Joe Leigh Simpson, MD, after leaving the Chair of the Ob/Gyn department at Baylor (Houston, TX), was moving to Florida to be involved in the creation of a new medical school. We now can confirm this move and offer further details: His new title is Executive Associate Dean for Academic Affairs at Florida International University College of Medicine, Miami, FL and we wish him very good luck in his new position.

Alan DeCherney, MD has also moved again. After being Chairman at Tufts (Boston, MA) and UCLA (Los Angeles, CA), he now is back at the East Coast as the new Chief of the Reproductive Biology Medicine and Biology Branch at NIH. He, in addition, of course, maintains his position as Editor-in-Chief of Fertility and Sterility, after receiving an impeccable evaluation (Buster JE. Fertil Steril 2006;86:790-4) upon completion of his 1st 7-year term, and a 2nd term appointment. We congratulate to both appointments! 